\title{
Clinical significance and characteristics of left-sided gallbladder: case series study of 10 patients
}

\author{
Doo-Ho Lee', Doojin Kim', Yeon Ho Park', Joo Seop Kim² \\ ${ }^{1}$ Department of Surgery, Gachon University Gil Medical Center, Gachon University School of Medicine, Incheon, Korea \\ ${ }^{2}$ Department of Surgery, Hallym University Kangdong Sacred Heart Hospital, Hallym University School of Medicine, Seoul, Korea
}

\begin{abstract}
Purpose: The aims of this case series study were to review the 10 patients who were diagnosed with left-sided gallbladder and analyze their anatomic variations in the bile duct, portal vein, and hepatic vessels.

Methods: In this case series study, 10 patients with left-sided gallbladder were retrospectively analyzed at 2 tertiary referral centers between April 2004 and May 2019.

Results: Mean age was 61.1 years; there were 7 women and 3 men. Ten patients underwent laparoscopic cholecystectomy for acute cholecystitis or symptomatic gallbladder stone. The mean operation time was 77.2 minutes. Three ports were used in laparoscopic cholecystectomy procedures. The mean postoperative hospital stay was 3.5 days, and there were no cases of surgery-related morbidity. Two patients had type 1 bile duct and 3 had type 3 bile duct (2 type 3B and 1 type 3A). The right posterior portal vein as the first branch of the main portal vein was observed in all patients. Segment IV branches of the left portal vein crossing over to the segment VIII territory were observed in 7 of the 10 patients.

Conclusion: Although left-sided gallbladder is a very rare disease, it is possible to diagnose it preoperatively and perform laparoscopic cholecystectomy safely by adjusting port position. The common important features of left-sided gallbladder include distribution of the left portal vein crossing over to the right side of the liver and increased size of the left portal vein. These variations may have important clinical implications in the management of hepatic resection including donor hepatectomy.

[Ann Surg Treat Res 2019;97(6):302-308]
\end{abstract}

Key Words: Anatomic variation, Hepatectomy, Laparoscopic cholecystectomy

\section{INTRODUCTION}

Left-sided gallbladder (LSGB) is a rare anomaly in which the gallbladder is located on the left side of the round ligament without situs inversus viscerum [1,2]. LSGB was first described by Hochstetter in 1856 [3]. The reported incidence of this anomaly is $0.1 \%-1.2 \%$ [2-5].

In LSGB, cystic duct and cystic artery anomalies are observed due to gallbladder location. The cystic duct is anastomosed to the left or right side of the common bile duct (CBD) or, rarely, anastomosed to the hepatic duct [2]. The cystic artery is usually long, originates on the right side of the $\mathrm{CBD}$, and courses to the left in front of the bile duct to the gallbladder [2].

LSGB is reportedly associated with right-sided round ligaments accompanied by abnormal intrahepatic portal venous branching, segment IV atrophy, congenital liver cysts, complete or partial situs inversus, a duplicate gallbladder, hypoplastic bile duct, and an anomalous pancreatobiliary ductal junction $[2,3,6$ 9]. In addition, anomalies such as the main bile duct lying in front of the first part of the duodenum and malrotation of the
Received September 9, 2019, Revised October 22, 2019,

Accepted November 5, 2019

Corresponding Author: Doojin Kim

Department of Surgery, Gachon University Gil Medical Center, Gachon University School of Medicine, 774-21 Namdong-daero, Namdong-gu, Incheon 21565, Korea

Tel: +82-32-458-3244, Fax: +82-32-458-3247

E-mail:drkdj@gilhospital.com

ORCID: https://orcid.org/0000-0002-2382-6724
Copyright (c) 2019, the Korean Surgical Society

(c) Annals of Surgical Treatment and Research is an Open Access Journal. All articles are distributed under the terms of the Creative Commons Attribution NonCommercial License (http://creativecommons.org/licenses/by-nc/4.0/) which permits unrestricted non-commercial use, distribution, and reproduction in any medium, provided the original work is properly cited. 
intestine, an anteduodenal portal vein, an annular pancreas, agenesis of the dorsal pancreas, polysplenia, and highly mobile right colon are associated with LSGB $[10,11]$.

Most cases of LSGB are diagnosed intraoperatively despite repeated radiological interventions [1]. The recognition of LSGB is important during cholecystectomy to avoid injury to the biliary tree [1]. When patients with LSGB undergo laparoscopic cholecystectomy, several modifications of the laparoscopic procedure may be effective, such as that the right-hand surgical ports should be placed to the left of the midline or preparation and clipping of the cystic duct should be performed as closely as possible to the infundibulum after the surrounding tissue is stripped down [2,12].

The aims of this case series study were to review the 10 patients who were diagnosed with LSGB and analyze their anatomic variations of the bile duct, portal vein, and hepatic vessels.

\section{METHODS}

\section{Patients and preoperative evaluation}

We retrospectively analyzed the clinical data of 10 patients who were diagnosed with LSGB in Gachon University Gil Medical Center and Kangdong Sacred Heart Hospital between April 2004 and May 2019. Eight patients were diagnosed with LSGB at the time of laparoscopic cholecystectomy. Two patients were diagnosed with LSGB by incidental findings on abdominal CT (Fig. 1). In the patients diagnosed with LSGB, the intrahepatic vascular and biliary systems, especially portal venous branching, were evaluated based on the ultrasound (US) imaging, abdominal CT, and/or magnetic resonance cholangiopancreatography (MRCP) findings (Fig. 2). One patient underwent endoscopic retrograde cholangiopancreatography for a CBD stone before the laparoscopic cholecystectomy. The right and left liver volumes were calculated respectively using PACS (pitcture archiving and communication system) software (INFINITT Healthcare, Seoul, Korea), excluding the volumes of vessels in preoperative abdominal CT scan. The study was approved by the Institutional Review Board at Gachon University Gil Medical Center (IRB No. 2019-259) and Kangdong Sacred Heart Hospital (IRB No. 2019-08-003). The informed consent was waived by the Institutional Review Board in both centers.

\section{Laparoscopic cholecystectomy for the LSGB patient}

Ten patients underwent laparoscopic cholecystectomy. When we performed conventional laparoscopic cholecystectomy, the second (for the left hand) and third (for the right hand; main working port) laparoscopic ports were inserted in the right upper quadrant area and epigastric area of the abdomen, respectively (Fig. 3A). In laparoscopic cholecystectomy for LSGB patients who diagnosed before the surgery, the second and third laparoscopic ports were inserted in the right upper and left upper quadrants of the abdomen, respectively (Fig. 3B). In laparoscopic cholecystectomy for LSGB patients who diagnosed during the surgery, the position of the third port can be determined after confirmation of the gallbladder's position through the second port (Fig. 4). Because of getting the proper angle for the right hand, the third port must be located in the left side of round ligament of LSGB patient. When the operators

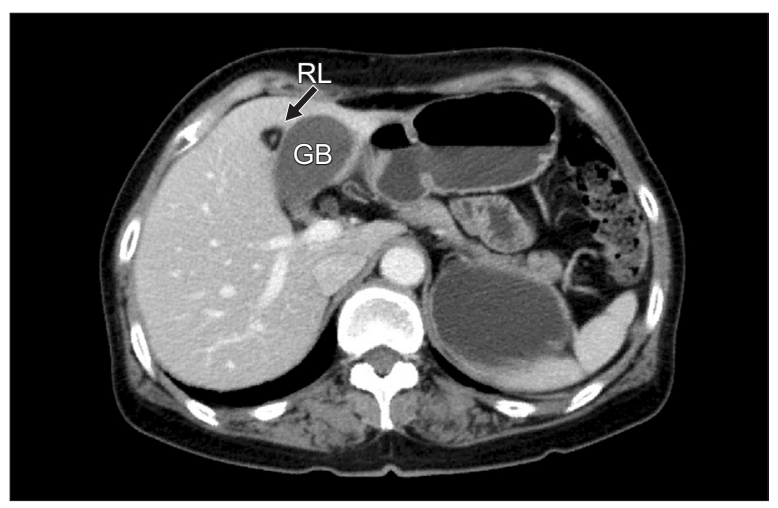

Fig. 1. Computed tomography image of left-sided gallbladder. The gallbladder (GB) was located on the left side of the round ligament (RL, black arrow) without situs inversus viscerum.
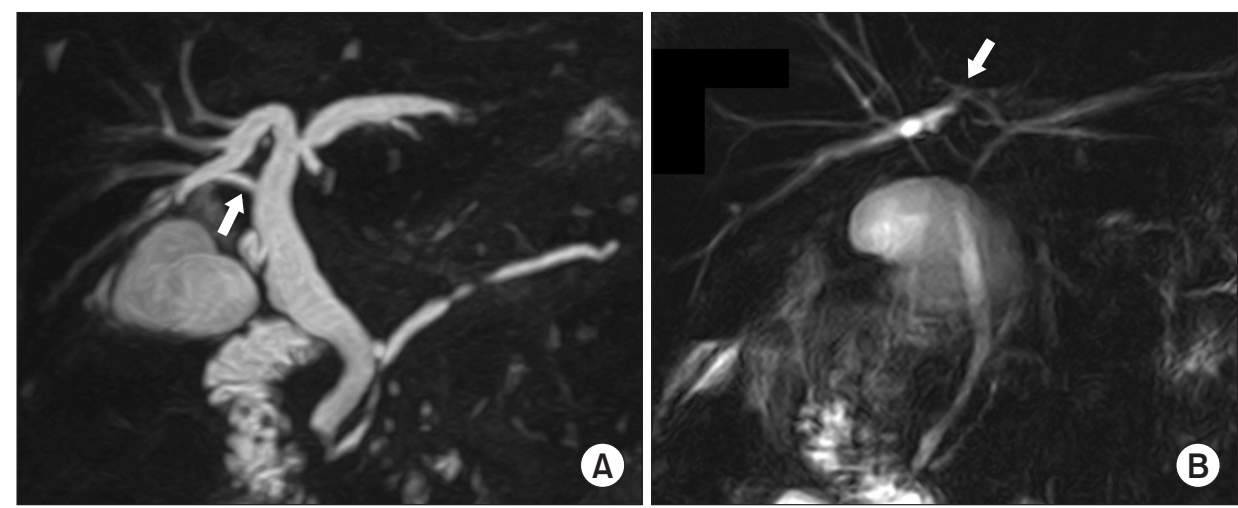

Fig. 2. Magnetic resonance cholangiopancreatography images of left-sided gallbladder. (A) Drainage of the right posterior segmental duct (white arrow) into the common hepatic duct (type 3B bile duct). (B) Drainage of the right posterior segmental duct (white arrow) into the left hepatic duct (type 3A bile duct). 
A

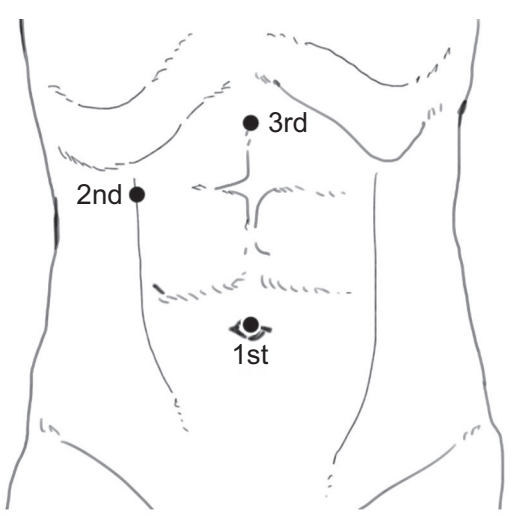

B

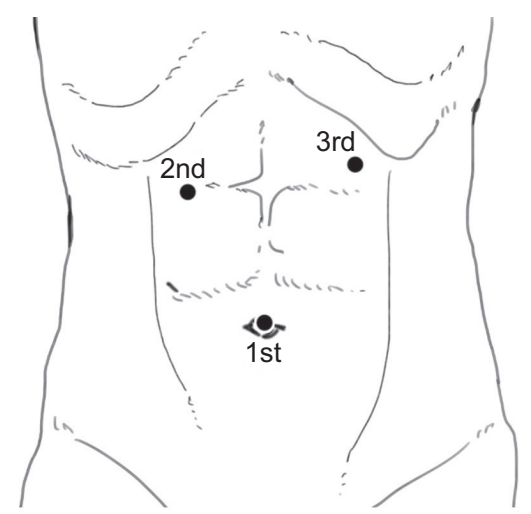

Fig. 3. Laparoscopic working port locations of laparoscopic cholecystectomy for left-sided gallbladder patients. (A) Laparoscopic working port location in conventional laparoscopic cholecystectomy. (B) Modified laparoscopic working port location of laparoscopic cholecystectomy for leftsided gallbladder patients.
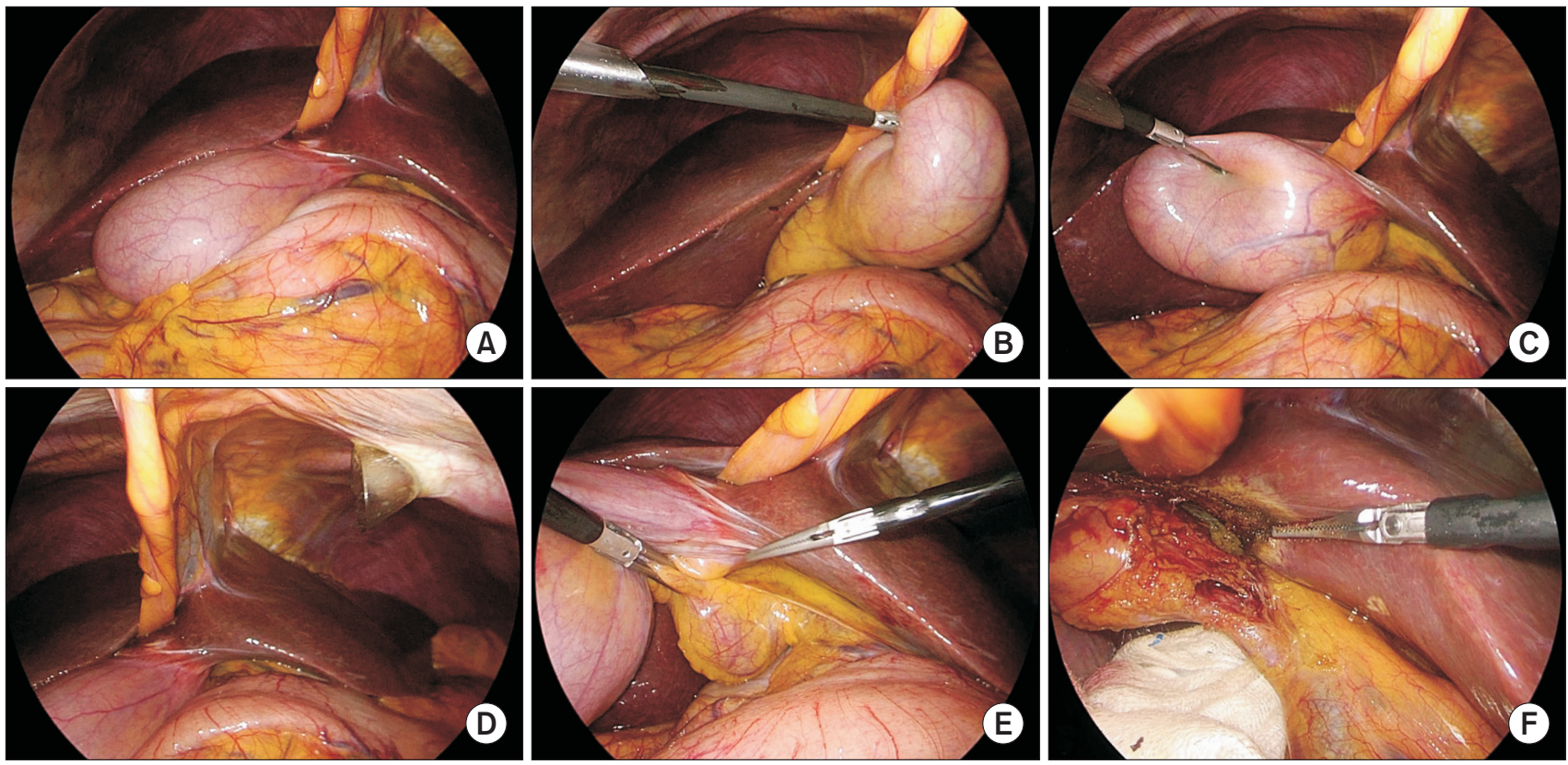

Fig. 4. Intraoperative images of left-sided gallbladder. (A) Laparoscopic view of left-sided gallbladder. (B) The second laparoscopic port (for the left hand) was inserted in the right upper quadrant of the abdomen. (C) The gallbladder bed was located to the left of the falciform ligament. (D) The third laparoscopic port (for the right hand) was inserted in the left upper quadrant of the abdomen. (E) Dissection of Calot's triangle. (F) The cystic duct and vessels of left-sided gallbladder.

could not get the critical view of safety during laparoscopic cholecystectomy for LSGB, we did top-down technique that removes the gallbladder from liver bed firstly.

\section{RESULTS}

\section{Patient characteristics and perioperative outcomes}

The patients' characteristics and perioperative outcomes are listed in Table 1. There were 7 women and 3 men; the median patient age (range) was 56.5 years (52-71 years), and the median body mass index (range) was $22.5 \mathrm{~kg} / \mathrm{m}^{2}\left(20.4-30.5 \mathrm{~kg} / \mathrm{m}^{2}\right)$. Ten patients underwent laparoscopic cholecystectomy for acute cholecystitis or symptomatic gallbladder stone. The median operation time (range) of laparoscopic cholecystectomy was 50 minutes (35-160 minutes). In 2 patients, operation time was relatively long due to severe gallbladder inflammation. Three ports were used in all laparoscopic cholecystectomy procedures. Seven patients underwent laparoscopic cholecystectomy with top-down technique. Median postoperative hospital stay (range) was 3 days (3-5 days); there were no cases of surgeryrelated morbidity. There were no cases of intraoperative cholangiography (IOC) procedure to prevent bile duct injury.

\section{Bile duct variation}

Five of 10 patients were evaluated with MRCP preoperatively for bile duct anatomy; of them, one also underwent ERCP due to CBD stones (Table 2). Two patients had a type 1 bile duct, in which a common hepatic duct (CHD) is formed by fusion of 
the right hepatic duct (RHD) and left hepatic duct (LHD) [13]. The RHD arises through fusion of the right anterior segmental duct, which drains anterior segments V and VIII, and the right posterior segmental duct (RPSD), which drains posterior segments VI and VII. Three patients had type 3 bile duct ( 2 with type 3B, 1 with type 3A) [13]. Type 3 bile duct, representing anomalous drainage of the RPSD, is subdivided into types $3 \mathrm{~A}$,
$3 \mathrm{~B}$, and $3 \mathrm{C}$ according to the drainage pattern: type $3 \mathrm{~A}$ drains into the LHD; type $3 \mathrm{~B}$ into the CHD; and type $3 \mathrm{C}$ into the cystic duct (Fig. 2).

\section{Portal vein variation}

The portal vein anatomy of 10 patients was evaluated preoperatively using abdominal CT (Table 2). Right posterior portal

Table 1. Characteristics and perioperative outcomes of patients with left-sided gallbladder

\begin{tabular}{|c|c|c|c|c|c|c|c|}
\hline Patient No. & Sex & Age (yr) & $\mathrm{BMI}\left(\mathrm{kg} / \mathrm{m}^{2}\right)$ & Diagnosis & $\begin{array}{l}\text { Operation } \\
\text { time (min) }\end{array}$ & $\begin{array}{l}\text { Postopera- } \\
\text { tive } \\
\text { hospital } \\
\text { stay (day) }\end{array}$ & Port No. \\
\hline 1 & Female & 57 & 22.8 & Symptomatic gallbladder stone & 60 & 3 & 3 \\
\hline 2 & Male & 63 & 22.7 & Acute cholecystitis & 140 & 4 & 3 \\
\hline 3 & Female & 56 & 22.2 & Acute cholecystitis & 160 & 3 & 3 \\
\hline 4 & Female & 74 & 21.9 & Acute cholecystitis & 45 & 4 & 3 \\
\hline 5 & Female & 77 & 23.9 & Acute cholecystitis & 75 & 5 & 3 \\
\hline 6 & Female & 52 & 20.4 & Acute cholecystitis & 45 & 4 & 3 \\
\hline 7 & Male & 53 & 30.5 & Symptomatic gallbladder stone & 50 & 3 & 3 \\
\hline 8 & Female & 52 & 22.4 & Symptomatic gallbladder stone & 50 & 3 & 3 \\
\hline 9 & Male & 71 & 22.3 & Acute cholecystitis & 85 & 3 & 3 \\
\hline 10 & Female & 56 & 24.4 & Symptomatic gallbladder stone & 35 & 3 & 3 \\
\hline
\end{tabular}

BMI, body mass index.

Table 2. Anatomic variations of the bile duct, portal vein, and hepatic vessels in patients with left-sided gallbladder

\begin{tabular}{|c|c|c|c|c|}
\hline $\begin{array}{l}\text { Patient } \\
\text { No. }\end{array}$ & Bile duct & Portal vein & Hepatic artery & Hepatic vein \\
\hline 1 & Type 1 & Type 3 & Normal anatomy & Type 2 \\
\hline 2 & Type 3B & Type 3 & Normal anatomy & Type 4 \\
\hline 3 & Type 3B & Type 3 & Normal anatomy & Type 4 \\
\hline 4 & - & Type 3 & Normal anatomy & Type 3 \\
\hline 5 & Type 3A & Type 3 & Normal anatomy & Type 2 \\
\hline 6 & Type 1 & Type 3 & Normal anatomy & Type 3 \\
\hline 7 & - & Type 3 & Normal anatomy & Type 2 \\
\hline 8 & - & Type 3 & Accessory left hepatic artery originating from the left gastric artery & Type 3 \\
\hline 9 & - & Type 3 & Accessory left hepatic artery originating from the left gastric artery & Type 2 \\
\hline 10 & - & Type 3 & Normal anatomy & Type 2 \\
\hline
\end{tabular}

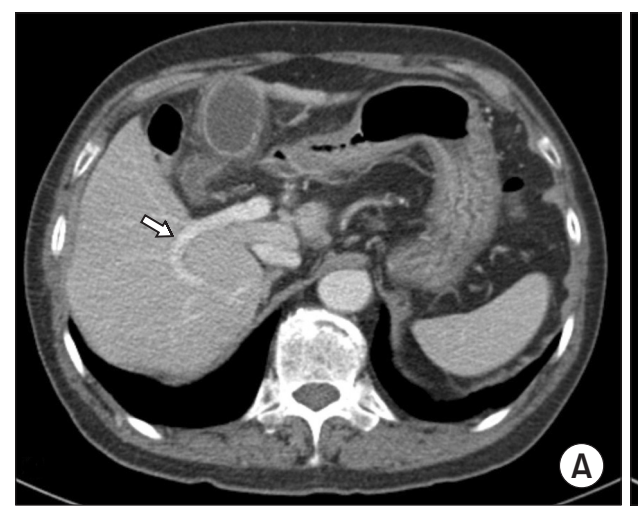

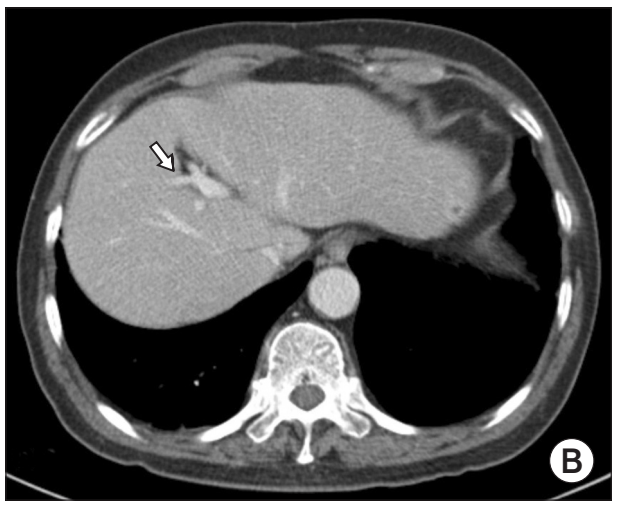

8
Fig. 5. CT images of portal vein variation. (A) Right posterior portal vein (white arrow) as the first branch of the main portal vein. (B) Segment IV branch (white arrow) of left portal vein crossing over to segment VIII territory. 
vein as the first branch of the main portal vein (type 3) was observed in all patients with LSGB (Fig. 5A) [14]. Segment IV branches of left portal vein crossing over to segment VIII territory were observed in 7 of the 10 patients with LSGB (Fig. 5B).

\section{Hepatic vessels variation}

An accessory left hepatic artery originating from the left gastric artery was observed in 2 of 10 patients, while normal hepatic artery anatomy was observed in rest (Table 2). Hepatic vein variation was evaluated preoperatively using abdominal CT and classified into 4 types according to superior right hepatic vein morphology [15]. Five of 10 patients were categorized into type 2 , in which a proximal confluence was formed by 2 tributaries running along the intersegmental border and draining nearby segments (V/VI or VI/VII) and a substantial tributary from segment VII close to the hepatocaval confluence was always present. Three of 10 patients were categorized into type 3 , in which the distal confluence was formed by 2 tributaries, creating posterolateral drainage of the complete segment VII and anteromedial drainage of the rest of the right side of the liver (segment VI, part of segments V and VIII). Two of 10 patients were categorized into type 4 , in which the distal confluence was formed by 2 tributaries from segments VII and VIII, respectively, while the accessory right hepatic veins, an inferior right hepatic vein alone, or an inferior right hepatic vein and a middle hepatic vein were always present.

\section{DISCUSSION}

In this case series, we reported 10 cases of LSGB in which all patients underwent laparoscopic cholecystectomy. Although LSGB is a rare anomaly, this study is one of the largest case series of it and is clinically significant because of the anatomic variations observed.

Most LSGB cases are diagnosed intraoperatively. In this study, 2 patients were diagnosed preoperatively according to abdominal CT findings, while the others were diagnosed intraoperatively. When we reviewed the abdominal CT findings, the 8 patients diagnosed intraoperatively could have been diagnosed preoperatively. Despite most of our patients being diagnosed intraoperatively, each laparoscopic procedure was performed safely.

When we performed conventional laparoscopic cholecystectomy, the second (for the left hand) and third (for the right hand) laparoscopic ports were inserted in the right upper quadrant and epigastric area of the abdomen. In laparoscopic cholecystectomy for LSGB patients, it was helpful for the surgeon to adjust the positions of the second and third laparoscopic ports in the left upper quadrant of the abdomen and epigastric area [1]. The location of the third working port was important to create a proper angle of dissection of Calot's tri- angle. The position of the third port can be determined after confirmation of the gallbladder's position through the second port. Also, it was more helpful to find cystic duct and cystic vessels that dissection of gallbladder bed was performed firstly, and then approaching the Calot's triangle was performed.

The IOC procedure, in which a catheter is introduced into the CBD for drainage of bile and injection of dye, can improve visualization of the bile duct anatomy and help the operator to avoid laparoscopic cholecystectomy induced damage $[16,17]$. Some studies have suggested that the most cost-effective treatment strategy for most patients with symptomatic cholelithiasis involved laparoscopic cholecystectomy with routine IOC, as IOC could help surgeon to visualize the biliary anatomy and detect unexpected CBD stones [18,19]. However, it remains debated whether IOC provides sufficient benefits in terms of its efficacy and safety to justify its routine application $[16,20]$. In this study, there were no cases of IOC procedure to prevent bile duct injury in LSGB patient who underwent laparoscopic cholecystectomy. Early 4 cases underwent MRCP preoperatively for bile duct anatomy; of them, one also underwent ERCP due to CBD stones. In recent 5 cases, the operator could distinguish the cystic duct and CBD during the operation because of accumulated experience of the surgeon.

Bile duct and portal vein variations were observed in 10 patients with LSGB. The right posterior portal vein as the first branch of the main portal vein (type 3) was observed in all patients with LSGB [14]. In addition, 7 patients had segment IV branches of the left portal vein crossing over to the segment VIII territory. These variations may have important clinical implications in the management of cholecystectomy as well as in the hepatic resection including donor hepatectomy [5,21]. Combined hepatic anomalies can complicate or prevent the resection of hepatobiliary malignancies. Thus, it is essential to establish a specific surgical plan after a thorough evaluation of the combined anomaly and tumor extent [22].

Patients with LSBG are generally considered unsuitable donors; however, several cases of successful living donor transplantation have been reported, in which portal vein anomalies were recognized [23-25]. Shimizu et al. [23] reported a patient with LSGB as a living liver donor in whom a right donor hepatectomy was performed in a patient with a right portal vein trifurcation anomaly. Hwang et al. [24] reported 3 cases of living donors with LSGB, among whom one had a bifurcating portal anomaly, intrahepatic right portal vein confluence, and extremely low bifurcation of the bile ducts, while the second donor had a trifurcating portal anomaly with a separate right posterior portal branch and replaced right posterior hepatic artery.

This case series found that the important feature of LSGB was the distribution of the left portal vein crossing over to the right side of the liver. In addition, a relative size reduction of the 
right portal vein, increased size of left portal vein, and portal vein supplying the left medial section extends to segment VIII of the liver, which was also observed in most patients.

In conclusion, although LSGB is a very rare disease, it is possible to diagnose it before surgery and safely perform laparoscopic cholecystectomy by adjusting the port positions. The common and important features of LSGB include the distribution of the left portal vein crossing over to the right side of the liver and increasing the size of the left portal vein. These variations may have important clinical implications in the management of hepatic resection including donor hepatectomy. To our knowledge, this is the largest case series of the clinical implications of LSGB and its anatomical variations.

\section{CONFLICTS OF INTEREST}

No potential conflict of interest relevant to this article was reported.

\section{ACKNOWLEDGEMENTS}

This work was supported by the Gachon University Gil Medical Center (Grant number: FRD2019-16).

\section{REFERENCES}

1. Zografos GC, Lagoudianakis EE, Grosomanidis D, Koronakis N, Tsekouras D, Chrysikos J, et al. Management of incidental left-sided gallbladder. JSLS 2009;13: 273-5.

2. Idu M, Jakimowicz J, Iuppa A, Cuschieri A. Hepatobiliary anatomy in patients with transposition of the gallbladder: implications for safe laparoscopic cholecystectomy. Br J Surg 1996;83:1442-3.

3. Nagai M, Kubota K, Kawasaki S, Takayama T, BandaiY, Makuuchi M. Are leftsided gallbladders really located on the left side? Ann Surg 1997;225:274-80.

4. Maetani Y, Itoh K, Kojima N, Tabuchi T, Shibata T, Asonuma K, et al. Portal vein anomaly associated with deviation of the ligamentum teres to the right and malposition of the gallbladder. Radiology 1998; 207:723-8.

5. Hsu SL, Chen TY, Huang TL, Sun CK, Concejero AM, Tsang LL, et al. Left-sided gallbladder: its clinical significance and imaging presentations. World J Gastroenterol 2007;13:6404-9.

6. Colovic R, Colovic N, Barisic G, Atkinson HD, Krivokapic Z. Left-sided gallbladder associated with congenital liver cyst. HPB (Oxford) 2006;8:157-8.

7. Rao PG, Katariya RN, Sood S, Rao PL. Situs inversus totalis with calculus cholecystitis and mucinous cystadenomas of ovaries. J Postgrad Med 1977;23:89-90.
8. Schachner A. Anomalies of the gall-bladder and bile-passages: with the report of a double gall-bladder and a floating gallbladder. Ann Surg 1916;64:419-33.

9. Hay SA, Soliman HE, Sherif HM, Abdelrahman AH, Kabesh AA, Hamza AF. Neonatal jaundice: the role of laparoscopy. J Pediatr Surg 2000;35:1706-9.

10. Korn O, Csendes A, Bastías J. Anomalies of extrahepatic biliary duct and gallbladder associated with intestinal malrotation: a case report. Surgery 1988;103:496-8.

11. Matsusue S, Kashihara S, Koizumi S. Pancreatectomy for carcinoma of the head of the pancreas associated with multiple anomalies including the preduodenal portal vein. Jpn J Surg 1984;14:394-8.

12. Hunter JG. Exposure, dissection, and laser versus electrosurgery in laparoscopic cholecystectomy. Am J Surg 1993;165:492-6.

13. Choi JW, Kim TK, Kim KW, Kim AY, Kim $\mathrm{PN}$, Ha HK, et al. Anatomic variation in intrahepatic bile ducts: an analysis of intraoperative cholangiograms in 300 consecutive donors for living donor liver transplantation. Korean J Radiol 2003;4: 85-90.

14. Koc Z, Oguzkurt L, Ulusan S. Portal vein variations: clinical implications and frequencies in routine abdominal multidetector CT. Diagn Interv Radiol 2007;13:7580.

15. De Cecchis L, Hribernik M, Ravnik D,
Gadzijev EM. Anatomical variations in the pattern of the right hepatic veins: possibilities for type classification. J Anat 2000;197 Pt 3:487-93.

16. Ding GQ, Cai W, Qin MF. Is intraoperative cholangiography necessary during laparoscopic cholecystectomy for cholelithiasis? World J Gastroenterol 2015;21:2147-51.

17. Kim NS, Jin HY, Kim EY, Hong TH. Cystic duct variation detected by near-infrared fluorescent cholangiography during laparoscopic cholecystectomy. Ann Surg Treat Res 2017;92:47-50.

18. Horwood J, Akbar F, Davis K, Morgan R. Prospective evaluation of a selective approach to cholangiography for suspected common bile duct stones. Ann R Coll Surg Engl 2010;92:206-10.

19. Brown LM, Rogers SJ, Cello JP, Brasel KJ, Inadomi JM. Cost-effective treatment of patients with symptomatic cholelithiasis and possible common bile duct stones. J Am Coll Surg 2011;212:1049-60.e1-7.

20. Lee DH, Ahn YJ, Lee HW, Chung JK, Jung IM. Prevalence and characteristics of clinically significant retained common bile duct stones after laparoscopic cholecystectomy for symptomatic cholelithiasis. Ann Surg Treat Res 2016;91:239-46.

21. Almodhaiberi H, Hwang S, Cho YJ, Kwon Y, Jung BH, Kim MH. Customized left-sided hepatectomy and bile duct resection for perihilar cholangiocarcinoma in a patient 
with left-sided gallbladder and multiple combined anomalies. Korean J Hepatobiliary Pancreat Surg 2015;19:30-4.

22. Kaneoka Y, Yamaguchi A, Isogai M, Harada T. Hepatectomy for cholangiocarcinoma complicated with right umbilical portion: anomalous configuration of the intrahepatic biliary tree. J Hepatobiliary Pancreat Surg 2000;7:321-6.
23. Shimizu T, Hayashi M, Inoue $Y$, Komeda $K$, Asakuma M, Hirokawa F, et al. Living-donor liver transplantation from donor with a left-sided gallbladder with portal vein anomaly. Transplantation 2012;94:e60-1.

24. Hwang S, Lee SG, Park KM, Lee YJ, Ahn CS, Kim KH, et al. Hepatectomy of living donors with a left-sided gallbladder and multiple combined anomalies for adult-to- adult living donor liver transplantation. Liver Transpl 2004;10:141-6.

25. Rocca JP, Rodriguez-Davalos MI, BurkeDavis M, Marvin MR, Sheiner PA, Facciuto $\mathrm{ME}$. Living-donor hepatectomy in rightsided round-ligament liver: importance of mapping the anatomy to the left medial segment. J Hepatobiliary Pancreat Surg 2006;13:454-7. 